\title{
Publisher's Note: Angular velocity of a spheroid log rolling in a simple shear at small Reynolds number [Phys. Rev. Fluids 1, 084203 (2016)]
}

\author{
J. Meibohm, F. Candelier, T. Rosén, J. Einarsson, F. Lundell, and B. Mehlig \\ (Received 3 January 2017; published 10 January 2017)
}

DOI: 10.1103/PhysRevFluids.2.019901

This paper was published online on 22 December 2016 with an incorrect title. The title should read as "Angular velocity of a spheroid log rolling in a simple shear at small Reynolds number." The title has been corrected as of 3 January 2017. 